Published in final edited form as:

Nature. ; 479(7373): 302-303. doi:10.1038/479302a.

\title{
Generations of longevity
}

\author{
Susan E. Mango \\ Department of Molecular and Cellular Biology, Harvard University, Cambridge, Massachusetts \\ 02138, USA.smango@mcb.harvard.edu
}

\section{Abstract}

The lifespan of some organisms can be extended by mutations that alter how DNA is packaged in their cells. A study reveals that this effect can last for generations, even in descendants that are genetically normal.

For decades, ageing was considered to be the result of progressive damage culminating in catastrophic breakdown. Yet similar animals can have vastly different lifespans - a barn owl can expect to live less than 8 years, a parrot more than 30 years — suggesting that there are mutable genetic pathways that control longevity. By studying small animals with short lifespans, such as the roundworm Caenorhabditis elegans, scientists have identified key pathways that can modulate ageing ${ }^{1}$. One of these involves the COMPASS complex (most probably an $\mathrm{H} 3 \mathrm{~K} 4 \mathrm{me} 3$ regulatory complex in $C$. elegans), an assembly of proteins that organizes DNA by chemically modifying a DNA-packaging protein called histone $\mathrm{H} 3$. Mutations in any of the three COMPASS subunits lead to defects in histone regulation and, in $C$. elegans, to a longer life ${ }^{2}$.

On page 365 of this issue, Greer et al. ${ }^{3}$ show that $C$. elegans worms have a memory of COMPASS mutations, even when they themselves carry normal, unmutated COMPASS components: great-grand-offspring and even great-great-grand-offspring live longer if their ancestors lacked COMPASS. In classical genetics, heritable changes in an organism's phenotype (its characteristics) that arise independently of DNA mutations are termed epigenetic inheritance, and have been a source of active research as scientists search for the causative mechanism. An attractive idea is that COMPASS generates an unknown molecular cue that can be inherited over multiple generations to restrict longevity.

Caenorhabditis elegans is not the only organism to show transgenerational effects in health and longevity. Examples can be found in other animals, including humans, and in plants. One of the earliest reported instances stems from studies in Sweden, where historical records revealed that the nutritional and smoking habits of paternal grandparents could influence their descendants' lifespan ${ }^{4}$. In the lab, transient exposure of rats to a high-sugar/low-protein diet leads to glucose intolerance ${ }^{5}$, and exposure of rats to noxious chemicals causes reduced fecundity ${ }^{6}$; these effects can last for generations ${ }^{7}$. Although such transgenerational, epigenetic effects have been documented for multiple species, the mechanism remains a mystery.

Greer et al. ${ }^{3}$ mated $C$. elegans worms that bore mutations in COMPASS subunits with wildtype males (Fig. 1). The resulting progeny, designated $\mathrm{F}_{1}$, were heterozygous for the 
mutation (that is, they inherited one mutant copy and one normal copy of the genes that encode COMPASS subunits) and were long-lived like their mutant mothers. Surprisingly, the $F_{1}$ animals gave rise to $F_{2}$ progeny, $F_{3}$ grand-offspring and $F_{4}$ great-grand-offspring that were genetically normal but phenotypically long-lived. The authors observed that this effect reverted at the $\mathrm{F}_{5}$ generation, when the animals' short lifespan was restored. When Greer et al. performed similar experiments to examine the influence of other regulators of histones, or of known modulators of longevity such as insulin receptors, they did not observe any transgenerational effects, indicating that the effect was specific to COMPASS.

The authors found that the long life of COMPASS mutants and their descendants required functional germ cells: $F_{3}$ animals that lacked germ cells, or that generated unfertilized eggs but no sperm, failed to live longer in response to ancestral COMPASS inactivation. The simplest interpretation of this result is that a COMPASS-dependent process occurs in germ cells to control lifespan, and that the flux of germ cells is important for its effects. However, an alternative possibility is that a functional germ line modulates other aspects of worm physiology. For example, it is known that $C$. elegans germ cells undergoing division control fat metabolism in the intestine, and that increased fat metabolism can extend life ${ }^{8}$. A similar non-autonomous effect may account for the germline dependence of COMPASS.

A question central to all transgenerational studies is how transient alterations in the environment or mutation can lead to long-term, multi-generational consequences. One attractive candidate mechanism has been methylation of DNA, a chemical modification that is associated with gene silencing. Most parental DNA methylation is removed in newly fertilized eggs, but some genes may retain methylated DNA over multiple generations, to serve as a transgenerational cue. This effect has been seen in mice, in which a region of DNA that controls coat colour can escape erasure of parental DNA methylation during embryonic development; the remaining degree of DNA methylation produces a range of fur colours ${ }^{6}$. But DNA methylation cannot be the only mechanism for transgenerational signalling, because $C$. elegans lacks DNA methylation altogether. Instead, Greer and colleagues' work ${ }^{3}$ implicates histone modifications - specifically, methylation of histone $\mathrm{H} 3$ at a particular amino acid (the H3K4me modification).

A crucial issue is whether $\mathrm{H} 3 \mathrm{~K} 4 \mathrm{me}$ itself is the inherited cue, or whether the modification in parental cells leads to downstream events that generate an epigenetic signal. Previous studies have shown that $\mathrm{H} 3 \mathrm{~K} 4 \mathrm{me}$ is established in the mother's germ line and inherited by the fertilized egg, where it is retained for at least the first few cell divisions ${ }^{9}$. One possibility, therefore, is that maintenance or interpretation of embryonic histone modifications affects adult worm longevity days later. Greer et al. ${ }^{3}$ show that their $C$. elegans COMPASS mutants lack $\mathrm{H} 3 \mathrm{~K} 4 \mathrm{me}$, and that restoration of $\mathrm{H} 3 \mathrm{~K} 4 \mathrm{me}$ correlates with a shorter life, suggesting that this histone modification is crucial for controlling longevity. But COMPASS can also function beyond individual genes, to influence the organization of chromosomes - in $C$. elegans, members of COMPASS associate with the dosage-compensation machinery, a complex of proteins that both controls the structure of the $\mathrm{X}$ chromosome and attenuates the expression of thousands of $\mathrm{X}$-linked genes ${ }^{10}$. Taken together, the evidence suggests that COMPASS may mediate transgenerational signalling directly, by controlling how genes and chromosomes are organized within cells. 
An alternative explanation is that COMPASS and $\mathrm{H} 3 \mathrm{~K} 4 \mathrm{me}$ activate gene expression, and that the resulting RNA or protein is inherited, rather than the H3K4me mark itself. Greer et al. identified genes that were misexpressed - that is, genes that were expressed when they shouldn't have been, and those that weren't expressed when they should have been -in COMPASS mutants, and found that many of the genes coded for proteins associated with longevity, growth or development. In line with the observed transgenerational effects ${ }^{3}$, these genes remained misexpressed in the $\mathrm{F}_{4}$ generation but reverted to normal expression levels in $\mathrm{F}_{5}$, and the expression of many of the genes was dependent on a functional germ line. An intriguing possibility is that the resulting RNAs are expressed in germline cells, where they could be placed in nascent oocytes (immature egg cells) and passed to the next generation. Although Greer et al. focused on messenger RNAs, small, non-coding RNAs (such as siRNAs, miRNAs and piRNAs) are also found in the C. elegans germ line and are probably inherited by the embryo. Small RNAs have regulatory roles in silencing gene expression, and could thereby shorten lifespan. Perhaps parental COMPASS is important for producing these non-coding RNAs.

One or a combination of the above explanations may account for the role of COMPASS in lifespan regulation, and it will be fascinating to learn how COMPASS induces transgenerational effects. Future studies will also be able to address whether COMPASS responds to environmental cues such as food, which would link the complex to the types of transgenerational influence that have been described in other animals. Finally, Greer and colleagues' study focused on the role of mothers, but in invertebrates it is clear that both mothers and fathers can signal to their descendants. It will be exciting to learn if COMPASS-dependent cues pass not only through the mother, but also through the father.

\section{References}

1. Kenyon CJ. Nature. 2010; 464:504-512. [PubMed: 20336132]

2. Greer EL, et al. Nature. 2010; 466:383-387. [PubMed: 20555324]

3. Greer EL. Nature. 2011; 479:365-371. [PubMed: 22012258]

4. Bygren LO, Kaati G, Edvinsson S. Acta Biotheor. 2001; 49:53-59. [PubMed: 11368478]

5. Ng S-F, et al. Nature. 2010; 467:963-966. [PubMed: 20962845]

6. Anway MD, Cupp AS, Uzumcu M, Skinner MK. Science. 2005; 308:1466-1469. [PubMed: 15933200]

7. Daxinger L, Whitelaw E. Genome Res. 2010; 20:1623-1628. [PubMed: 21041414]

8. Wang MC, O’Rourke EJ, Ruvkun G. Science. 2008; 322:957-960. [PubMed: 18988854]

9. Li T, Kelly WG. PLoS Genet. 2011; 7:e1001349. [PubMed: 21455483]

10. Pferdehirt RR, Kruesi WS, Meyer BJ. Genes Dev. 2011; 25:499-515. [PubMed: 21363964] 


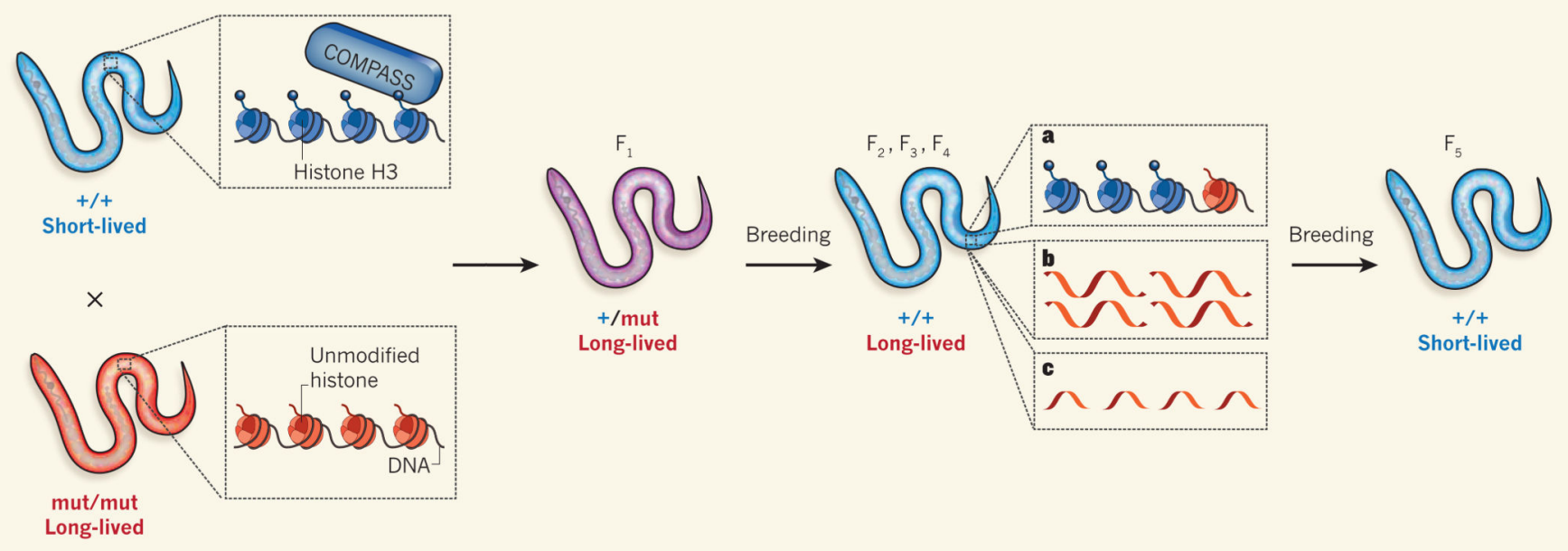

Figure 1. Transgenerational effects of COMPASS mutations on longevity The COMPASS protein complex organizes DNA by chemically modifying a DNApackaging protein called histone H3. Wild-type Caenorhabditis elegans worms (blue) are short-lived compared with mutant worms lacking COMPASS (red). Greer et al..$^{3}$ crossed wild-type $C$. elegans (which have two copies of the normal COMPASS-encoding gene; $+/+$ ) with mutant $C$. elegans (which have two mutant copies of the COMPASS-encoding gene; $\mathrm{mut} / \mathrm{mut}$ ), and obtained long-lived, heterozygous (+/mut) worms (designated as $\mathrm{F}_{1}$ ). The next three generations $\left(\mathrm{F}_{2}-\mathrm{F}_{4}\right)$ of the worms were genetically normal $(+/+)$, but were still long-lived. The mechanism underpinning the longevity of $F_{2}$ to $F_{4}$ is unknown, but may reflect: a, the presence of unmodified histones at some locations of the genome; $\mathbf{b}$, altered expression of protein-encoding messenger RNAs; or $\mathbf{c}$, altered expression of small, noncoding RNAs that regulate gene expression. $\mathrm{F}_{5}$ worms were genetically normal, and not long-lived. 